\title{
Supporting Information \\ Host contact dynamics shapes richness and dominance of pathogen strains
}

\author{
Francesco Pinotti ${ }^{1}$, Éric Fleury $^{2}$, Didier Guillemot ${ }^{3}$, Pierre-Yves Böelle $^{1}$, Chiara \\ Poletto $^{1}$ \\ 1 Sorbonne Université, INSERM, Institut Pierre Louis d'Épidémiologie et de \\ Santé Publique,Paris,France \\ 2 INRIA, Paris, France \\ 3 Inserm, UVSQ, Institut Pasteur, Université Paris-Saclay, Biostatistics, Biomath- \\ ematics, Pharmacoepidemiology and Infectious Diseases (B2PHI), Paris, France.
}

\section{Multi-strain model with heterogeneous recovery classes}

In order to test the robustness of our results to the case in which more detailed and realistic ingredients are accounted for, we consider the case in which the recovery rate is heterogeneous across individuals. This mimics a relevant aspect of bacterial colonization, as in e.g. S. aureus case. In [1] individuals were subdivided in three main classes with very different carriage duration, i.e. noncarriers, intermittent carriers and persistence carriers. Similarly, we assumed three classes of individuals characterized by different recovery probability per time step, i.e. $\mu^{(1)}=1, \mu^{(2)}=1.3 \cdot 10^{-3}$ and $\mu^{(3)}=6.9 \cdot 10^{-4}$. Nodes are distributed in the three classes with probability $p^{(1)}=0.5, p^{(2)}=0.3$ and $p^{(3)}=0.2[1]$. Notice that individuals in the first class recover immediately after a single time step. Recovery rates are chosen so that the average carriage period matches the value used in the main paper.

\section{References}

[1] Qiuzhi Chang, Marc Lipsitch, and William P. Hanage. Impact of Host Heterogeneity on the Efficacy of Interventions to Reduce Staphylococcus 
aureus Carriage. Infect Control Hosp Epidemiol, 37(2):197-204, February 2016. 